\title{
Analysis of the crystal structure of an active MCM hexamer
}

\author{
Justin M Miller ${ }^{\dagger}$, Buenafe T Arachea ${ }^{\dagger}$, Leslie B Epling, Eric J Enemark* \\ Department of Structural Biology, St Jude Children's Research Hospital, Memphis, \\ United States
}

\begin{abstract}
In a previous Research article (Froelich et al., 2014), we suggested an MCM helicase activation mechanism, but were limited in discussing the ATPase domain because it was absent from the crystal structure. Here we present the crystal structure of a nearly full-length MCM hexamer that is helicase-active and thus has all features essential for unwinding DNA. The structure is a chimera of Sulfolobus solfataricus $\mathrm{N}$-terminal domain and Pyrococcus furiosus ATPase domain. We discuss three major findings: 1) a novel conformation for the A-subdomain that could play a role in MCM regulation; 2) interaction of a universally conserved glutamine in the $\mathrm{N}$-terminal Allosteric Communication Loop with the AAA+ domain helix-2-insert (h2i); and 3) a recessed binding pocket for the MCM ssDNA-binding motif influenced by the h2i. We suggest that during helicase activation, the h2i clamps down on the leading strand to facilitate strand retention and regulate ATP hydrolysis. DOI: 10.7554/eLife.03433.001
\end{abstract}

\begin{abstract}
*For correspondence: eric. enemark@stjude.org

tThese authors contributed equally to this work
\end{abstract}

Competing interests: The authors declare that no competing interests exist.

Funding: See page 10

Received: 21 May 2014 Accepted: 28 August 2014 Published: 29 September 2014

Reviewing editor: Michael $\mathrm{R}$ Botchan, University of California, Berkeley, United States

(c) Copyright Miller et al. This article is distributed under the terms of the Creative Commons Attribution License, which permits unrestricted use and redistribution provided that the original author and source are credited.

\section{Introduction}

Hexameric MCM rings act as the replicative DNA helicase (Bochman and Schwacha, 2008; Ilves et al., 2010), encircling the leading strand DNA template at the replication fork (Fu et al., 2011). Mcm2-7 complexes are loaded (reviewed in Remus and Diffley, 2009) to encircle double-stranded DNA (dsDNA) via a 'gate' between Mcm2 and Mcm5 (Bochman and Schwacha, 2007, 2008; Costa et al., 2011) to yield a double hexamer (Evrin et al., 2009; Remus et al., 2009) that does not unwind DNA. During helicase activation, the Dbf4-dependent Cdc7 kinase (DDK) and cyclin-dependent kinases (CDKs) drive recruitment of Cdc45 and the GINS complex (Labib, 2010). These factors stimulate the Mcm2-7 ATPase and helicase (IIves et al., 2010) and with Mcm2-7 form the CMG complex (Cdc45-Mcm2-7-GINS), the active replicative helicase (Moyer et al., 2006; Bochman and Schwacha, 2008; Ilves et al., 2010). Following activation, two Mcm2-7 helicases encircle single-stranded DNA (ssDNA) and translocate independently (Yardimci et al., 2010), 3' $\rightarrow 5^{\prime}$, on the leading strand DNA template (Fu et al., 2011) with the ATPase domain leading (McGeoch et al., 2005).

Our previous crystal structure of the Pyrococcus furiosus $\mathrm{MCM} N$-terminal domain $\left(P f \mathrm{MCM}_{N}\right)$ bound to ssDNA revealed an MCM single-stranded binding motif (MSSB) that binds ssDNA (Froelich et al., 2014). Our discussion of an MSSB role in helicase activation invoked action of the AAA+ (reviewed in Duderstadt and Berger, 2013) ATPase domain to translocate DNA, but we could not discuss specifically how the MSSB was affected by the AAA+ domain because it was not present in the PfMCMN:SSDNA structure. Now, we present the crystal structure of a helicase-active MCM hexamer to reveal a novel conformation for the A-subdomain that could play a role in MCM regulation and how the AAA+ helix2-insert is tethered to the $\mathrm{N}$-terminal domain to create a recessed binding pocket for the MSSB.

\section{Results and discussion}

We identified a chimera of the N-terminal domain of Sulfolobus solfataricus (Sso) and the AAA+ domain of PfMCM, Sso-PfMCM (Figure 1A) with a robust DNA unwinding activity (Figure 1B; Figure 1figure supplement 1). We present an analysis of the crystal structure of the Sso-PfMCM hexamer bound to $\mathrm{Mg} / \mathrm{ADP}$ (Table 1). 


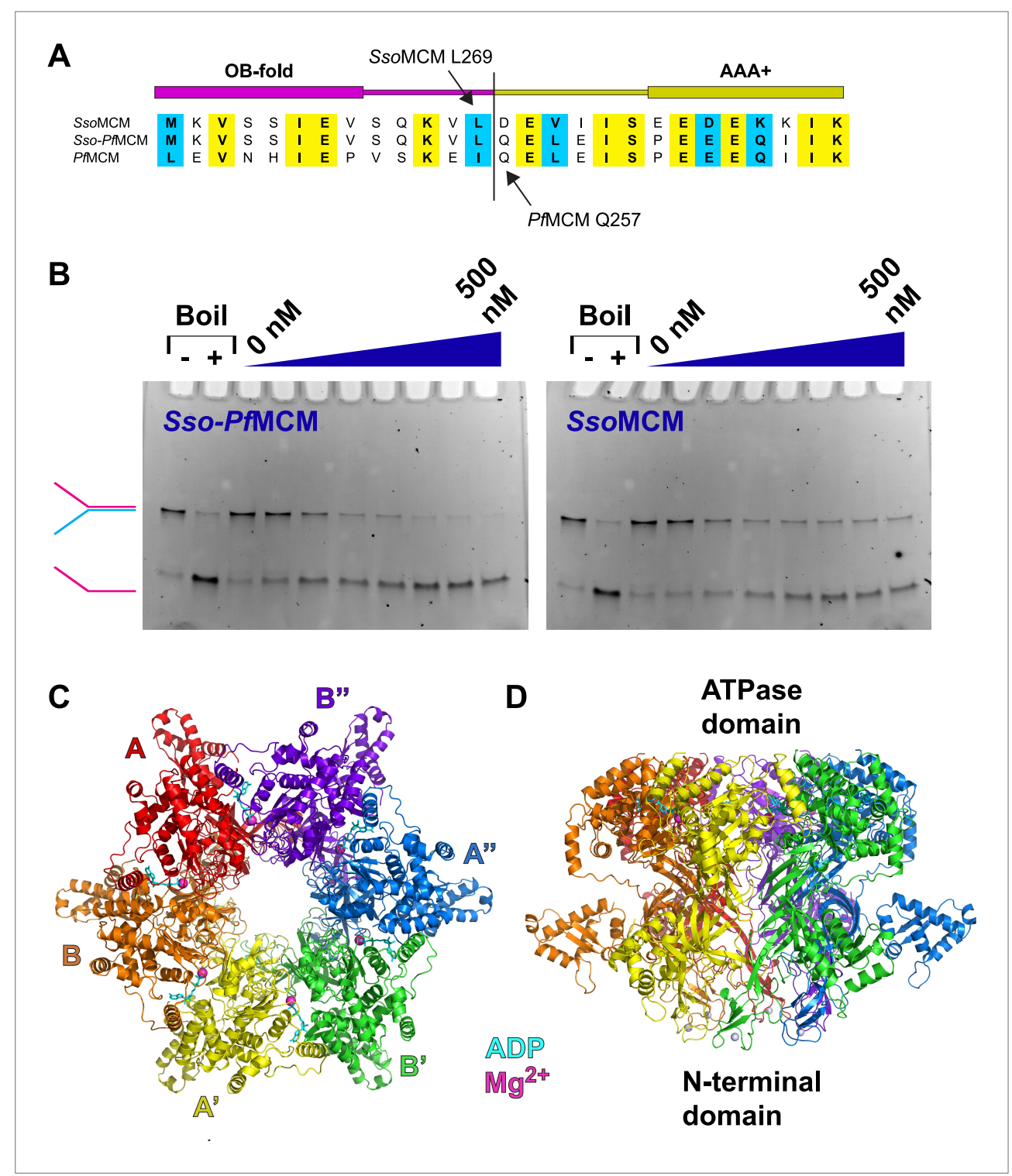

Figure 1. Properties of Sso-PfMCM. (A) Sequence alignment showing the construction of the Sso-PfMCM chimera. The N-terminal domain of SsoMCM (residues 1-269, top sequence) was fused to the AAA+ domain of PfMCM (starting at residue 257, bottom sequence) to yield the chimera (middle sequence). (B) The Sso-PfMCM chimera shows enhanced unwinding activity when compared to wild-type SsoMCM. Helicase reactions were performed at $69^{\circ} \mathrm{C}$ for 60 min with a Y-shaped DNA substrate with a $5^{\prime}$-fluorescein label on one strand. Unwinding reactions were in the presence of $4 \mathrm{mM}$ ATP and contained 0,50, 100, 150, 200, 300, 400, or $500 \mathrm{nM}$ protein. Views of the Sso-PfMCM hexamer crystal structure parallel (C) and perpendicular (D) to the central channel with each subunit uniquely colored. The magnesium ions are magenta spheres, and ADP molecules are shown as cyan stick. (C) View down the crystallographic threefold axis with the unique and symmetry-derived chains labeled. The ATPase domains are projected out of the page. (D) View perpendicular to the channel axis. The ATPase domains are located at the top, and the N-terminal domains are located at the bottom. The $\mathrm{Zn}$ ions are light grey spheres at the bottom. DOI: 10.7554/eLife.03433.002

The following figure supplements are available for figure 1:

Figure supplement 1. MCM catalyzed DNA unwinding visualized by gel electrophoresis. DOI: 10.7554/eLife.03433.003

Figure supplement 2. The relative positions of the $\mathrm{N}$ - and $\mathrm{C}$-terminal domains in Sso-PfMCM significantly differ from previous monomeric crystal structures.

DOI: 10.7554/eLife.03433.004

Figure supplement 3. Activity and structure of PfMCM ${ }_{\text {AAA }}$. DOI: 10.7554/eLife.03433.005 
Table 1. Data collection and refinement statistics

\begin{tabular}{|c|c|c|}
\hline & Sso-PfMCM:MgADP Hexamer & PfMCM $_{\mathrm{AAA}}:$ MgADP Double-octamer \\
\hline \multicolumn{3}{|l|}{ Data collection } \\
\hline Space group & $\mathrm{P}_{3}$ & P1 \\
\hline \multicolumn{3}{|l|}{ Cell dimensions } \\
\hline$a, b, c(\AA)$ & $118.902,118.902,199.317$ & $124.956,127.082,128.025$ \\
\hline$\alpha, \beta, y\left(^{\circ}\right)$ & $90,90,120$ & $71.852,72.819,80.392$ \\
\hline Resolution $(\AA ̊)$ & $50-2.70(2.80-2.70)$ & $50-3.80(3.94-3.80)$ \\
\hline$R_{\text {sym }}$ & $0.107(0.750)$ & $0.169(0.429)$ \\
\hline$|/ \sigma|$ & $14.8(1.79)$ & $8.3(2.41)$ \\
\hline Completeness (\%) & $99.8(98.3)$ & $98.9(97.0)$ \\
\hline Redundancy & $6.8(5.0)$ & $3.3(2.6)$ \\
\hline \multicolumn{3}{|l|}{ Refinement } \\
\hline Resolution $(\AA)$ & $50-2.70(2.80-2.70)$ & $50-3.80(3.94-3.80)$ \\
\hline No. reflections & $39,044 / 1976(2042 / 123)$ & $69,126 / 3486(6206 / 357)$ \\
\hline$R_{\text {work }} / R_{\text {free }}$ & $0.263 / 0.295(0.360 / 0.353)$ & $0.301 / 0.314(0.367 / 0.368)$ \\
\hline \multicolumn{3}{|l|}{ No. atoms } \\
\hline Protein & 9432 & $2429(1 / 16$ of ASU) \\
\hline ADP & 54 & 27 (1/16 of ASU) \\
\hline ions & 10 & $1(1 / 16$ of $\mathrm{ASU})$ \\
\hline Water & 0 & 0 \\
\hline \multicolumn{3}{|l|}{$B$-factors } \\
\hline Protein & 60 & 91 \\
\hline ADP & 118 & 72 \\
\hline ions & 75 & 81 \\
\hline Water & N/A & $\mathrm{N} / \mathrm{A}$ \\
\hline \multicolumn{3}{|l|}{ R.m.s. deviations } \\
\hline Bond lengths (Å) & 0.010 & 0.010 \\
\hline Bond angles $\left({ }^{\circ}\right)$ & 1.488 & 1.597 \\
\hline
\end{tabular}

\section{MCM:Mg/ADP hexamer crystal structure}

The Sso-PfMCM hexamer (Figure 1C-D; Video 1, 0:00) forms a ring with a channel large enough to accommodate double-stranded DNA (dsDNA) (see 'Materials and methods'). The Sso-PfMCM structure therefore could mechanistically represent the structure of the MCM hexamer prior to loading (no DNA), after it loads to encircle dsDNA, or after its activation (encircling ssDNA). The two-tiered structure is consistent with electron microscopy studies (Chong et al., 2000; Pape et al., 2003; GomezLlorente et al., 2005; Costa et al., 2006; Bochman and Schwacha, 2007; Remus et al., 2009; Costa et al., 2011). The N-terminal tier has three subdomains, A-C (Fletcher et al., 2003; Liu et al., 2008; Froelich et al., 2014) with the A-subdomains in a different orientation (Figure 2A; Video 1, 0:20; see below) than observed previously. The relative positions of $\mathrm{MCM}_{\mathrm{N}}$ and $\mathrm{MCM}_{\mathrm{AAA}}$ differ considerably compared to monomeric and filament MCM crystal structures (Brewster et al., 2008; Bae et al., 2009; Slaymaker et al., 2013) (Figure 1-figure supplement 2). This difference is needed to prevent serious clashes that would occur among the ATPase domains.

\section{A-subdomain is in a different orientation than in previous structures}

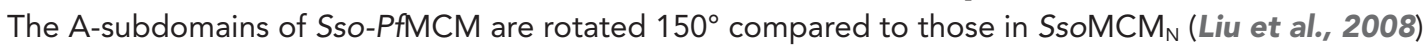
(Figure 2A). The previously identified A-subdomain conformation seems fully possible in our present structure (see Video 1, 0:20), and we suggest that different A-subdomain conformations might play a role in MCM regulation. A similar A-subdomain rotation has been suggested for Methanothermobacter 


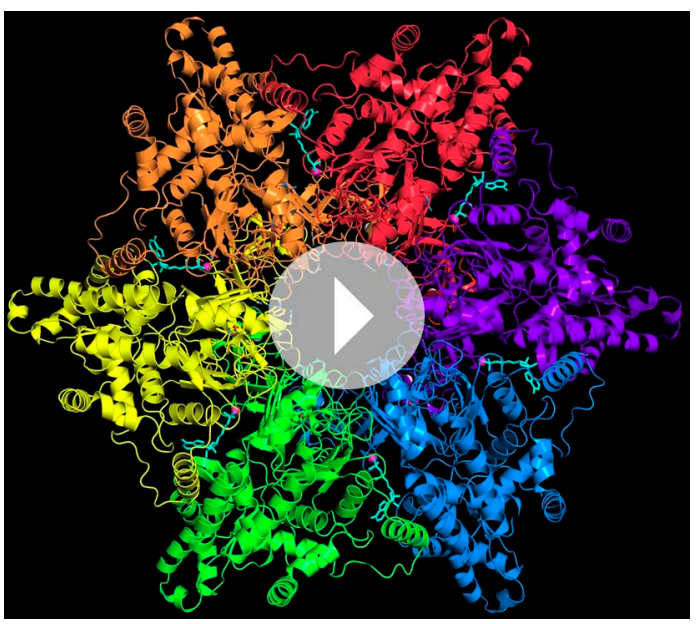

Video 1. Crystal structure details for Sso-PfMCM. The video illustrates the arrangement of the subunits in the hexamer and the positions of the subdomains. The A-subdomain conformation is animated to transform to that observed in other crystal structures of $\mathrm{MCM}_{\mathrm{N}}$ to illustrate how they differ. The different A-subdomain conformations correlate with the conformation of a proline (P104) at the junction between the A- and C-subdomains. The relative position of the $\mathrm{mcm} 5-$ bob 1 mutation is noted. Several central channel modules are highlighted, including the ps1 $\beta$, h2i, $\beta$-turn, MSSB, and the interaction of ACL $\mathrm{Q} 198$ with the h2i. The ATPase site is compared to that of papillomavirus E1 (Enemark and Joshua-Tor, 2006), and several key residues are highlighted for MCM. The MSSB location is shown in a surface representation to illustrate that it sits at a recessed binding pocket where the ssDNA (green) of the aligned PfMCM :ssDNA crystal structure (Froelich et al., 2014) would position snugly.

DOI: 10.7554 /eLife.03433.007

$\mathrm{N}$-terminal serine/threonine-rich domain by DDK (Sheu and Stillman, 2006) that serves both inhibitory and facilitating roles in replication (Sheu and Stillman, 2010).

\section{Central channel modules}

Several modules are directed into the central channel where they could interact with encircled DNA (Figure 2B; Video 1, 1:19). The pre-sensor-1- $\beta$-hairpin (ps1 $\beta$ ) projects a universally conserved lysine, K785, that is essential for unwinding by SsoMCM (McGeoch et al., 2005). The helix-2-insert (h2i), required for helicase activity in MtMCM (Jenkinson and Chong, 2006), prominently directs R734 and W741 into the central channel. These residues are conserved in a family-specific fashion for Mcms (Video 1, 1:32). The h2i and ps1 $\beta$ are located further from the $\mathrm{N}$-terminal domain than predicted by monomeric crystal structures (Figure 1-figure supplement 2) due to interdomain differences (see above). The h2i projects further into the channel than the ps1 $\beta$ and appears to divide the $A A A+$ and $N$-terminal DNA-binding regions. As viewed in Figure $2 B$, the ps1 $\beta$ and the $\mathrm{h} 2 \mathrm{i}$ direct their putative DNA-binding residues above the $\mathrm{h} 2 \mathrm{i}$, while the MSSB is below the $\mathrm{h} 2 \mathrm{i}$. The $\mathrm{h} 2 \mathrm{i}$ creates a DNA-binding pocket at the MSSB where ssDNA was observed previously (Froelich et al., 2014). In SsoMCM, alanine mutants of lysine residues in this pocket (K129A and K194A) show severe DNA-binding and unwinding defects (Pucci et al., 2004). While the MSSB pocket of Sso-PfMCM appears poised to bind ssDNA in the fashion observed previously (Figure 2C), some remodeling of $\mathrm{h} 2 \mathrm{i}$ side-chains or the ssDNA would be necessary to avoid clashes, particularly involving F737. 
A

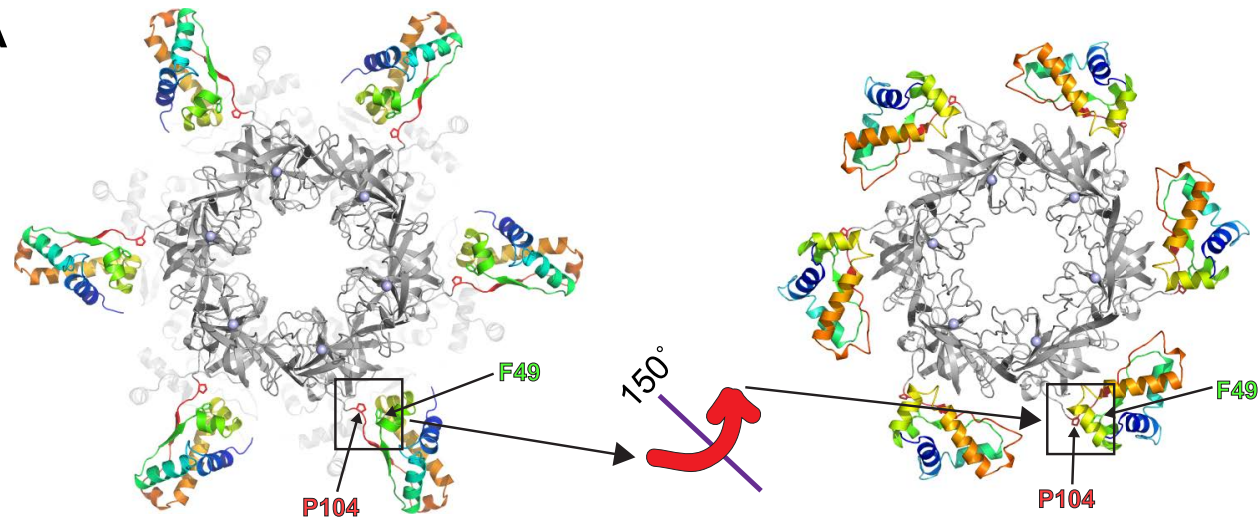

Sso-PfMCM

SsoMCM $_{\mathrm{N}}$ PDB:2VL6

B

C SSDNA from aligned

PMCMN:SSDNA (PDB: 4POG)
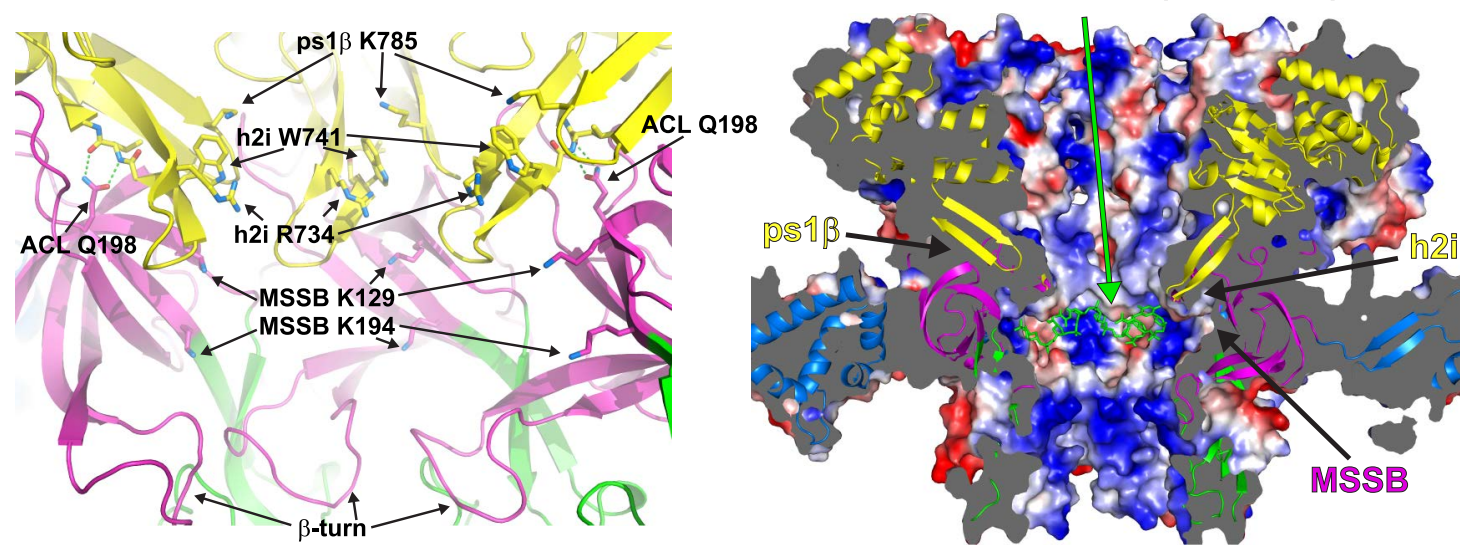

D
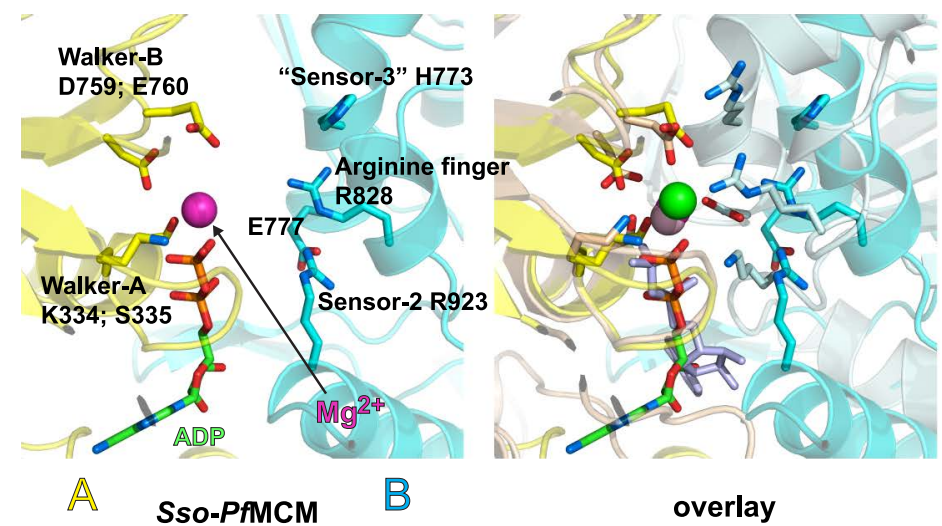

A Sso-PfMCM

overlay

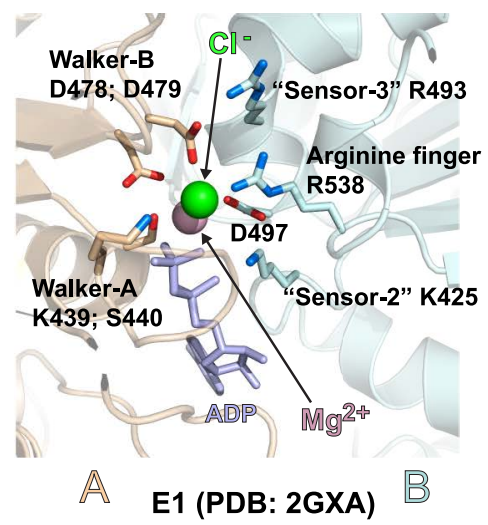

Figure 2. Sso-PfMCM crystal structure details. (A) The A-subdomains (rainbow) of Sso-PfMCM adopt a unique conformation that is rotated $150^{\circ}$ compared to other crystal structures of hexameric $\mathrm{MCM}_{N}$ such as SsoMCM $($ Liu et al., 2008). The distinct conformations correlate with the conformation of P104 that is located at the junction between the A- and C-subdomains (boxed). In both conformations, P104 packs against the aromatic residue F49. (B) The modules of the central channel. The cartoon is colored with the AAA+ domain in yellow, the Zn-binding B-subdomain in green, and the OB-fold C-subdomain in magenta. The ps1 $\beta$ projects a conserved lysine, K785, into the channel. This lysine packs against W741 of the h2i, which sits adjacent to R734 of the h2i. The MSSB is recessed and sits below the h2i and above the $\beta$-turn. The N-terminal domain is tethered to the h2i by a universally conserved glutamine in the ACL, Q198. (C) Surface representation of Sso-PfMCM colored by electrostatic potential. The surface is clipped Figure 2. Continued on next page 
Figure 2. Continued

with a vertical plane through the center to illustrate the central channel features. A cartoon representation of the protein with select modules labeled is colored as in Figure 2B with the helical A-subdomain in blue. The MSSB sits at a recessed pocket. The ssDNA from the aligned PfMCMN:SsDNA structure (Froelich et al., 2014) would be snugly positioned in this pocket. (D) Comparison of the Sso-PfMCM ATPase site (left) with that of E1 (Enemark and Joshua-Tor, 2006, right). The Walker-A and Walker-B residues of one subunit (yellow) are positioned at the left side of the site while three positively charged residues of the adjacent subunit (cyan) line the right side of the site. An acidic residue of the cyan subunit sits below the site. Based on the superposition (middle), we predict that the MCM subunits need to approach each other more closely to generate a competent ATPase site. DOI: 10.7554/eLife.03433.008

The following figure supplement is available for figure 2:

Figure supplement 1. Cartoon model showing a role for an interaction between the $\mathrm{N}$-terminal $\mathrm{ACL}$ conserved glutamine and the AAA+ h2i during helicase activation.

\section{Allosteric Communication Loop}

In each subunit of our Sso-PfMCM hexamer crystal structure, residues 198-212 comprise a conserved loop of the OB-fold that projects towards the $h 2 \mathrm{i}$ of the same subunit and the ps1 $\beta$ of an adjacent subunit. This loop has been termed the 'Allosteric Communication Loop' (ACL) (Barry et al., 2009) due to its predicted proximity to the AAA+ domain and its observed influence on unwinding (Sakakibara et al., 2008; Barry et al., 2009). Although the ATPase domain alone is sufficient to generate DNA unwinding in SsoMCM (Barry et al., 2007; Pucci et al., 2007) and in Aeropyrum pernix MCM (Atanassova and Grainge, 2008), several mutants located on this loop show unwinding defects (Sakakibara et al., 2008; Barry et al., 2009). In our hexamer structure, the proximity of the ACL to the h2i and ps1 $\beta$, highly significant AAA+ modules (see above), strongly supports an ACL role in $\mathrm{N}$ - and C-terminal domain communication. The ACL position near the ps $1 \beta$ of an adjacent subunit is also consistent with previous studies (Barry et al., 2009).

We tested the DNA unwinding activity of PfMCM $\mathrm{AAA}_{\mathrm{AA}}$ to compare with that of the Sso-PfMCM chimera to explore interdomain communication. We found $P A M C M_{A A A}$ had a negligible unwinding activity (Figure 1-figure supplement 3). The inactivity of $P A M C M_{A A A}$ could result from attributes identified in the PfMCM ${ }_{\text {AAA }}$ double-octamer crystal structure (see 'Materials and methods'). Specifically, the nonhexameric ring architecture or the alternative topology for the helix-2-insert region, both of which we consider artifacts of the $\mathrm{N}$-terminal truncation, could fully explain the lack of unwinding by this domain. Thus, although $S$ soMCM $M_{N}$ does enhance the unwinding activity of $P f M C M M_{A A A}$, this could simply be due to enforcing a hexameric architecture or by disallowing the unusual h2i topology in favor of the canonical topology.

We next examined the Sso-PfMCM structure for interdomain interactions that could be conserved in native proteins. The structure reveals a fully conserved glutamine, Q198, of the ACL interacts with the main-chain amide atoms of the h2i (Figure 2B; Video 1, 1:50). This interaction could occur in any MCM protein because the constituent atoms are fully conserved. The Q198:h2i interaction is not required for DNA unwinding because no unwinding defects are observed for the Q198A mutant (Figure 1-figure supplement 1), and in the corresponding alanine mutant of MtMCM (Sakakibara et al., 2008). We suggest that the glutamine-h2i interaction functions prior to unwinding to lock the $\mathrm{h} 2 \mathrm{i}$ in a holding position. The movement of DNA proposed previously to facilitate initial strand separation (Froelich et al., 2014) could occur by ATP-hydrolysis-driven inward movement of the h2i bound to DNA (Figure 2-figure supplement 1). After the h2i modules reach the position observed in our structure, they would be locked in place by interaction with Q198. This would tighten the grasp on one strand while the opposing strand exits the open Mcm2/5 gate (Bochman and Schwacha, 2007; Costa et al., 2011). With the h2i modules fixed in this position, the ATPase sites would be unable to adopt a productive hydrolysis conformation because each ATPase site is fundamentally tied to h2i position, potentially to prevent further ATPase activity during an important activation event. ATP hydrolysis inhibition by fixed $\mathrm{h} 2 \mathrm{i}$ is directly analogous to inhibition of ATP hydrolysis in $\$ 12$ P4, an RNA-translocating hexamer, by cross-linked RNA-binding loops (Kainov et al., 2008).

\section{ATPase active site}

The ATPase active site structurally resembles the ATPase site of the AAA+ helicase E1 (Enemark and Joshua-Tor, 2006) (Figure 2D, Video 1, 2:04) with Walker-A/B residues (Abrahams et al., 1994; 
Neuwald et al., 1999) of one subunit, and three positively charged residues of the adjacent subunit. The three positive residues consist of sensor-2 (Neuwald et al., 1999), the arginine finger (Neuwald et al., 1999), and residues that we classify as sensor-3 (Enemark and Joshua-Tor, 2006). Although not typical for AAA+ proteins, the placement of sensor- 2 in an ATPase site among Walker-A/B residues of the neighboring subunit (in trans) was predicted for MCM proteins in defining AAA+ Clade 7 (Erzberger and Berger, 2006). Biochemical experiments (Moreau et al., 2007) and MCM structurebased predictions (Bae et al., 2009) are also consistent with this arrangement. Based on comparison of the ATPase site with the tight 'ATP-like' configuration of E1 (Enemark and Joshua-Tor, 2006), we expect MCM subunits must approach more closely to generate a competent ATPase site. In this state, the $\mathrm{h} 2 \mathrm{i}$ and $\mathrm{ps} 1 \beta$ are predicted to move upward in the view shown in Figure 2-figure supplement 1 to increase the distance between the $A C L$ and the $p s 1 \beta$ as identified by DEER-spectroscopy (Barry et al., 2009). ATP-hydrolysis would drive the $h 2 \mathrm{i} / \mathrm{ps} 1 \beta$ downward to translocate one ssDNA strand with expected polarity and orientation (McGeoch et al., 2005) while the complementary strand is excluded from the ring (Fu et al., 2011). Our present structural findings cannot differentiate several mechanistic details such as hydrolysis order or timing (reviewed in Singleton et al., 2007). We speculate that the MCM helicase unwinds DNA with helically-arranged h2i/ps1 $\beta$ modules analogous to E1 (Enemark and Joshua-Tor, 2006) and Rho (Thomsen and Berger, 2009), but the six non-identical subunits of Eukaryotic Mcm2-7 could operate asymmetrically during unwinding. Indeed, the ATPase modules of the different Mcm2-7 subunits show distinct roles and specialization during different functional stages (Coster et al., 2014; Kang et al., 2014). Elucidation of how the AAA+ domain interacts with DNA in an unwinding conformation will help reveal more details of the MCM unwinding mechanism.

\section{Materials and methods}

\section{Cloning, mutagenesis, expression, and purification}

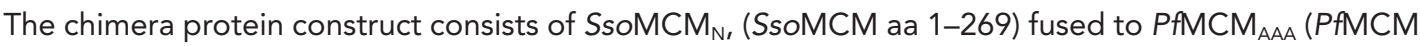
aa 257-361/729-966 = aa 257-966 with its intein, aa 362-728, removed). It corresponds to a fulllength MCM protein lacking the short (aa 967-1049) C-terminal helix-turn-helix domain (Aravind and Koonin, 1999) that is dispensible for unwinding activity in MtMCM (Jenkinson and Chong, 2006) and in SsoMCM (Barry et al., 2007). All expression constructs were prepared as $\mathrm{N}$-terminal $\mathrm{His}_{6}$-SUMO fusions. The original SUMO vector was the generous gift of Dr Christopher D Lima (Mossessova and Lima, 2000). The PfMCM gene contains an intein, aa 362-728 (Yoshimochi et al., 2008) in the helix-2-insert region of ATPase domain. We genetically removed the intein by sequentially cloning two fragments (amplified from $P$. furiosus genomic DNA, ATCC) incorporating a silent Notl mutation at the junction. This plasmid served as the PCR template to generate constructs of

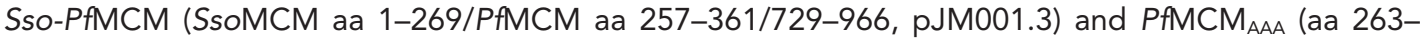
361/729-966, pEE021.1 = crystallized construct; and PfMCM aa 252-361/729-1049 = construct of unwinding experiments). The Sso-PfMCM chimera construct was generated by overlap extension of PCR fragments encoding the N-terminal domain of SsoMCM (amplified from S. solfataricus genomic

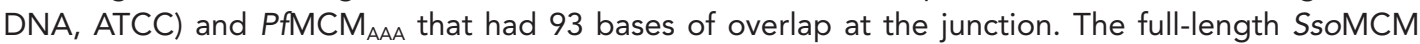
expression construct (pEE045.1) was prepared via PCR amplification of the full SsoMCM gene. The Q198A mutant (pJM005.5) was generated by site-directed mutagenesis of pJM001.3. DNA sequencing verified the integrity of the coding region of all constructs. Proteins were purified as described previously (Froelich et al., 2014), including removal of the SUMO tag by digestion by Ulp1 protease (the Ulp1 protease plasmid was the generous gift of Dr Christopher D Lima) (Mossessova and Lima, 2000).

\section{Crystallization, data-collection, structure-solution, and refinement}

Prior to crystallization, purified Sso-PfMCM was dialyzed into buffer containing $25 \mathrm{mM} \mathrm{HEPES,} \mathrm{pH} \mathrm{7.6;}$ $10 \mathrm{mM} \mathrm{NaCl}$; and $5 \mathrm{mM} \mathrm{Mg}(\mathrm{OAc})_{2}$. Crystals of Sso-PfMCM with Mg/ADP grew by hanging drop by mixing $2 \mu \mathrm{l}$ of protein:ADP solution $(10.8 \mathrm{mg} / \mathrm{ml}$ Sso-PfMCM; $5 \mathrm{mM}$ ADP) and $2 \mu \mathrm{l}$ of well solution (100 mM HEPES, pH 7.6; 350 mM MgCl$; 3 \%$ (wt/vol) PEG 3350). Crystals were cryoprotected by quickly passing through a 1:3 ethylene glycol:well solution and flash frozen in liquid nitrogen. Data were collected at SER-CAT beamline 22-ID. Data were collected at $1.0 \AA$ wavelength in $0.25^{\circ}$ oscillations for $112.5^{\circ}$ at a temperature of $100 \mathrm{~K}$. All data were scaled and integrated using the HKL-2000 software package (Otwinowski and Minor, 1997) to 2.70 Å resolution. 
The structure was solved in space group $\mathrm{P}_{3}$ by the program Phaser (McCoy et al., 2007), which

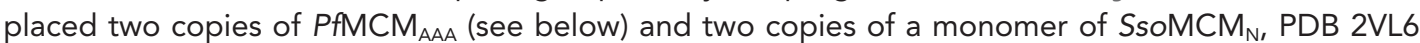
(Liu et al., 2008), in a single hexamer on a crystallographic threefold axis. Overall, the unit cell contains two nearly sixfold symmetric hexamers offset by a strong NCS translation of [1/3, 2/3, 1/2] (Patterson peak height $50 \%$ of origin). Initial electron density maps revealed a clear misplacement of the helical A-subdomain, which was corrected by Phaser (McCoy et al., 2007) by using separate search models for the A-subdomain and the B/C-subdomains. The initial electron density map was greatly improved by multi-crystal electron density averaging in the AAA+ domain region with the program Dmmulti (Cowtan, 1994) by implementing twofold averaging of Sso-PfMCM and 16-fold averaging of PfMCM ${ }_{A A A}$. The model was refined at various stages with CNS (Brunger et al., 1998; Brunger, 2007), phenix (Afonine et al., 2012), and refmac5 (Vagin et al., 2004) and manually improved with Coot (Emsley and Cowtan, 2004). The final refinement was carried out in CNS (Brunger et al., 1998; Brunger, 2007). A Ramachandran plot calculated by Procheck (Laskowski et al., 1993) indicated the following statistics: core: 917 (86.8\%); allowed: 124 (11.7\%); generously allowed: 15 (1.4\%); disallowed: 0 (0\%). Figures were prepared with PyMOL (Schrodinger, 2010), Molscript (Kraulis, 1991), and Raster3D (Merritt and Bacon, 1997 ).

Crystallographic datasets for crystals grown in the presence of several nucleotide cofactors (ADP, AMP-PNP, ATP-yS, ADP-AIF $)_{x}$ were collected. All were strongly isomorphic with crystals grown with $\mathrm{Mg} / \mathrm{ADP}$, and no evidence of a $\mathrm{Y}-\mathrm{PO}_{4}$ or its analog was ever detected in resulting electron density maps. We therefore conclude that all crystal datasets adopt a highly similar structure most consistent with an ADP-bound state and that the $\mathrm{Y}-\mathrm{PO}_{4} /$ analog hydrolyzes over the course of crystallization or is crystallographically disordered.

The crystal structure of PfMCM $\mathrm{AAA}_{\text {was }}$ wivotal in obtaining the crystal structure of Sso-PfMCM (above), and we therefore include the details of its structure determination. However, the resolution of PfMCM $\mathrm{AAA}_{\text {, }}(3.80 \AA)$ limits the overall detail of the structure. Crystals of PfMCM $\mathrm{AAA}_{\text {with }} \mathrm{Mg} / \mathrm{ADP}$ grew by hanging drop by mixing $2 \mu \mathrm{l}$ protein/Mg/ADP $(6 \mathrm{mg} / \mathrm{ml} ; 5 \mathrm{mM} \mathrm{ADP;} 50 \mathrm{mM} \mathrm{MgCl} ; 18 \mathrm{mM}$ HEPES, pH 7.6; $180 \mathrm{mM} \mathrm{NaCl} ; 4.5 \mathrm{mM} \beta$-mercaptoethanol) with $2 \mu$ well solution (50 mM sodium cacodylate, $\mathrm{pH}$ 6.0; 50 mM magnesium acetate; 30\% MPD; 5\% glycerol). A Crystal was flash frozen in liquid nitrogen and data were collected at SER-CAT beamline 22 -BM at $1.0 \AA$ wavelength in $0.5^{\circ}$ oscillations for $360^{\circ}$ at a temperature of $100 \mathrm{~K}$. The data were scaled and integrated using the HKL-2000 software package (Otwinowski and Minor, 1997) to $3.80 \AA$ resolution. A weak molecular replacement solution was obtained with the program Phaser (McCoy et al., 2007), which placed 16 monomers of the AAA+ portion of PDB 4FDG (Slaymaker et al., 2013) as a double-octamer. The MCM complex is not presumed to adopt an octameric assembly in vivo, but we note that a hypothetical hexamer generated by removing two adjacent subunits from the octameric ring would correspond to an open hexameric ring with an opening large enough to permit entry of B-form dsDNA. Initial electron density maps were greatly improved by 16-fold NCS-averaging and solvent flattening with the program Resolve (Terwilliger, 2000, 2004), which revealed obvious side-chain positions. The sequence was assigned to the structure based upon the location of selenium positions (7 per subunit) for a selenomethionine derivative in an NCS-averaged anomalous difference Fourier map generated with the Resolve-improved phases, and by alignment with the $1.90 \AA$ resolution structure of a monomeric MCM homolog (Bae et al., 2009). The selenomethionine derivative was expressed in B834(DE3) cells (EMD Millipore, Darmstadt, Germany) in LeMaster's media (Hendrickson et al., 1990), and anomalous difference data were collected at SER-CAT beamline 22-ID at $0.97915 \AA$ A wavelength in $0.5^{\circ}$ oscillations for $260^{\circ}$ at a temperature of $100 \mathrm{~K}$. The data were scaled and integrated using the HKL-2000 software package (Otwinowski and Minor, 1997) to $4.0 \AA$ resolution. The anomalous signal was too weak to generate starting phases, but readily identified the selenium positions by an NCS-averaged anomalous difference fourier map generated with the Resolve-improved molecular replacement phases (see above). The structure was refined with a strict 16-fold NCS protocol in CNS (Brunger et al., 1998; Brunger, 2007) and manually improved with Coot (Emsley and Cowtan, 2004). Following refinement of the higher resolution structure of Sso-PfMCM (above), the coordinates were updated, and the h2i region was rebuilt. The structure was subjected to coordinate and group B-factor refinement with strict 16-fold NCS in CNS (Brunger et al., 1998; Brunger, 2007) to yield the final model. The h2i is folded differently than in other AAA+ proteins to mediate a $\beta$-sheet interface with the ps $1 \beta$ of a subunit in the other octamer. Twofold symmetric dimers are arranged around an eightfold symmetry axis to yield approximate D8-symmetry. A total of 16 of these $\beta$-sheet interfaces occur around the ring. This $h 2 i$ structure is not compatible with the position of the N-terminal domain seen in Sso-PfMCM, and it is 
therefore almost certainly an artifact of removing the $\mathrm{N}$-terminal domain in the PfMCM $\mathrm{AAA}_{\text {c }}$ construct. The precise sequence registry for these $\mathrm{h} 2 \mathrm{i}$ residues is not clearly defined, and the residues have been modeled as poly-alanine in our best assessment of the polypeptide direction. A Ramachandran plot calculated by Procheck (Laskowski et al., 1993) indicated the following statistics: core: 213 (74.7\%); allowed: 56 (19.6\%); generously allowed: 8 (2.8\%); disallowed: 8 (2.8\%).

\section{Helicase assay}

All unwinding experiments were performed with a Y-shaped DNA substrate with a 55-mer doublestranded region, a 50-mer poly-dT 3'-arm, and a 30-mer $5^{\prime}$-arm with a fluorescein label at the $5^{\prime}$ - end. The substrate was prepared by annealing a $5^{\prime}$-fluorescein-labeled oligonucleotide $\left(5^{\prime}\right.$-TTGAACCA CCCCCTTGTTAAATCACTTCTACTTGCATGCCTGCAGGTCGACTCTAGAGGATCCCCGGGT ACCGAGCTCGAATTCG-3' with an unlabeled oligonucleotide (5'- CGAATTCGAGCTCGGTACCC GGGGATCCTCTAGAGTCGACCTGCAGGCATGCAAGTTTTTTTTTTTTTTTTTTTTTTTTTTTTTTTTTTTT TTTTTTTTTTTTTTTTT-3'), Sigma-Aldrich, St. Louis, MO). The 85-mer oligonucleotide was identical to a previously published substrate that had been annealed to M13 plasmid ssDNA to study SsoMCM unwinding (Pucci et al., 2004). Helicase activity assays were prepared in $20 \mu$ reaction mixtures with $25 \mathrm{mM}$ HEPES (pH = 7.6), $100 \mathrm{mM} \mathrm{Na(OAc),} 5 \mathrm{mM} \mathrm{Mg}(\mathrm{OAc})_{2}, 4 \mathrm{mM}$ ATP, and $3.7 \mathrm{nM}$ fluorescein-labeled DNA substrate. For protein concentration titrations, the protein concentration ranged from 0 to $500 \mathrm{nM}$ (monomer), and reactions were incubated at $69^{\circ} \mathrm{C}$ for $60 \mathrm{~min}$. For time-course experiments, $500 \mathrm{nM}$ protein was incubated at $69^{\circ} \mathrm{C}$ for 1 to $90 \mathrm{~min}$. Prior to the addition of ATP to initiate unwinding, time points were incubated at $69^{\circ} \mathrm{C}$ for $5 \mathrm{~min}$ to allow for thermal equilibration. For all samples, reactions were stopped by the addition of $5 \mu \mathrm{l}$ of loading buffer containing $40 \%$ (vol/vol) glycerol, $5 \%$ (wt/vol) sodium dodecyl sulfate (SDS), and $50 \mathrm{mM}$ ethylenediaminetetraacetic acid (EDTA), and a $20 \mu \mathrm{l}$ aliquot was loaded on a 4-20\% 1× TBE gradient PAGE gel (Biorad, Berkeley, CA) and run at $150 \mathrm{~V}$ for $90 \mathrm{~min}$. Gel imaging was performed with a Fuji LAS-4000 using a 15-min exposure time and a SYBR-Green filter.

\section{Definition of central channel axis, mathematical analysis}

We conclude that the central channel of our structure of the Sso-PfMCM hexamer is sufficiently large to accommodate dsDNA based on a channel radius minimum of $12.7 \AA$ for its polyalanine model. For comparison, the polyalanine model of BPV E1:ssDNA (encircles ssDNA; Enemark and Joshua-Tor, 2006; Lee et al., 2014) has a minimum radius of $6.1 \AA$ (PDB: 2GXA, hexamer 1, Enemark and JoshuaTor, 2006); topoisomerase I:dsDNA (tightly encircles dsDNA) has a minimum radius of $7.5 \AA$ (PDB: 1 A35, Redinbo et alo, 1998); and PCNA (encircles dsDNA but can slide) has a minimum radius of $16.0 \AA$ (PDB: 1PLQ, Krishna et al., 1994). Based on these comparisons, the channel diameter in our structure of Sso-PfMCM is large enough to accommodate dsDNA, but might not slide over dsDNA as readily as PCNA. The details of these calculations are provided below.

Our analysis of the central channel of a ring requires a definition of the channel axis. We define this axis as the rotation axis for least-squares permutation of the subunits. For Sso-PfMCM, this axis coincides with a crystallographic threefold axis. For straightforward analysis, we use the following procedure, which is general, to produce a transformed PDB coordinate file with the channel axis coincident with the $[0,0, Z]$ axis of the standard PDB coordinate system. First, the coordinates of a full ring molecule were translated to place the center-of-mass on the origin of the standard PDB coordinate system with the program MOLEMAN (Kleywegt, 1997). Second, the least-squares rotation that explicitly permutes the subunits (for example, chains ABCDEF onto chains BCDEFA in a hexamer) was calculated by LSOMAN (Kleywegt, 1996), expressed in polar angles (Omega $=\mathrm{O}, \mathrm{Phi}=\mathrm{P}, \mathrm{Chi}=\mathrm{C})$. These polar angles were used to transform the origin-shifted coordinates in two successive polar rotation operations with the program MOLEMAN (Kleywegt, 1997). The first polar rotation was by $(0,0,-P)$, and the second was by $(90,90,-O)$. Subsequently, the distance of any atom to the channel axis can be calculated from its $X$ and $Y$ coordinates in the transformed PDB coordinate file as the square root of $\left(X^{2}+Y^{2}\right)$. For Topoisomerase I (PDB id: 1A35, Redinbo et al., 1998), the channel axis was defined as the least-squares helical axis of the bound dsDNA, and this axis was transformed to coincide with the $[0,0, Z]$ axis of the PDB coordinate system as described above.

\section{Acknowledgements}

Data were collected at Southeast Regional Collaborative Access Team (SER-CAT) 22-ID and 22-BM beamlines at the Advanced Photon Source, Argonne National Laboratory. Supporting institutions may 
be found at www.ser-cat.org/members.html. We are grateful to SER-CAT staff for experimental support. Use of the Advanced Photon Source was supported by the U. S. Department of Energy, Office of Science, Office of Basic Energy Sciences, under Contract No. W-31-109-Eng-38.

\section{Additional information}

Funding

\begin{tabular}{lll} 
Funder & Grant reference number & Author \\
\hline $\begin{array}{l}\text { American Lebanese Syrian } \\
\text { Associated Charities (ALSAC) }\end{array}$ & & Eric J Enemark \\
\hline $\begin{array}{l}\text { National Institute of General } \\
\text { Medical Sciences }\end{array}$ & R01GM098771 & Eric J Enemark \\
\hline Cancer Center Support Grant & 5 P30 CA021765 & Eric J Enemark \\
\hline
\end{tabular}

The funders had no role in study design, data collection and interpretation, or the decision to submit the work for publication.

Author contributions

JMM, Acquisition of data, Analysis and interpretation of data, Drafting or revising the article; BTA, Acquisition of data, Analysis and interpretation of data, Drafting or revising the article, Contributed unpublished essential data or reagents; LBE, Drafting or revising the article, Contributed unpublished essential data or reagents; EJE, Conception and design, Analysis and interpretation of data, Drafting or revising the article

\section{Additional files}

Supplementary file

- Supplementary file 1. ClustalW sequence alignment of several MCM proteins in MSF format. Portions of this alignment are shown throughout Video 1. The Sso-PfMCM residue numbers in the alignment run consecutively, which is consistent with database numbering for the SsoMCM portion. For the PfMCM portion, database numbers can be obtained from the aligned PfMCM sequence. The intein (residues 362-728) was manually removed from the PfMCM sequence for the alignment. Thus, for residues of $P f M C M$ in the alignment with sequence numbers greater than 361, 367 must be added to agree with database (intein-containing) sequence numbering. Residue numbering throughout the manuscript and Video 1 agrees with the database numbering for the respective portions.

DOI: 10.7554/eLife.03433.010

Major dataset

The following dataset/s was/were generated:

ratase, license,

\begin{tabular}{|c|c|c|c|c|}
\hline Author(s) & Year & Dataset title & Dataset ID and/or URL & $\begin{array}{l}\text { and accessibility } \\
\text { information }\end{array}$ \\
\hline $\begin{array}{l}\text { Miller JM, Arachea BT, } \\
\text { Epling LB, Enemark EJ }\end{array}$ & 2014 & $\begin{array}{l}\text { Crystal structure of an active } \\
\text { MCM hexamer (SsoPfMCM } \\
\text { hexamer) }\end{array}$ & $\begin{array}{l}\text { http://www.pdb.org/ } \\
\text { pdb/explore/explore. } \\
\text { do?structureld=4R7Y }\end{array}$ & $\begin{array}{l}\text { Publicly available } \\
\text { at RCSB Protein } \\
\text { Data Bank. }\end{array}$ \\
\hline $\begin{array}{l}\text { Miller JM, Arachea BT, } \\
\text { Epling LB, Enemark EJ }\end{array}$ & 2014 & PfMCM-AAA double-octamer & $\begin{array}{l}\text { http://www.pdb.org/ } \\
\text { pdb/explore/explore. } \\
\text { do?structureld=4R7Z }\end{array}$ & $\begin{array}{l}\text { Publicly available } \\
\text { at RCSB Protein } \\
\text { Data Bank. }\end{array}$ \\
\hline
\end{tabular}

The following previously published dataset/s was/were used:

Database, license, and accessibility

\begin{tabular}{|c|c|c|c|c|}
\hline Author(s) & Year & Dataset title & Dataset ID and/or URL & information \\
\hline Enemark EJ, Joshua-Tor L & 2006 & $\begin{array}{l}\text { Crystal structure of } \\
\text { papillomavirus E1 hexameric } \\
\text { helicase with ssDNA and } \\
\text { MgADP }\end{array}$ & $\begin{array}{l}\text { http://www.pdb.org/ } \\
\text { pdb/explore/explore. } \\
\text { do?structureld=2gxa }\end{array}$ & $\begin{array}{l}\text { Publicly available } \\
\text { at RCSB Protein } \\
\text { Data Bank. }\end{array}$ \\
\hline
\end{tabular}


Redinbo MR, Stewart L, Kuhn P, Champoux JJ, Hol WG

Krishna TS, Kong XP, Gary S, Burgers PM, Kuriyan J

Liu W, Pucci B, Rossi M

Pisani FM, Ladenstein RLiu W,

Pucci B, Rossi M, Pisani FM, Ladenstein $\mathrm{R}$

Slaymaker IM, Fu Y, Toso DB Ranatunga N, Brewster A Forsburg SL, Zhou ZH, Chen XS
1998 Human topoisomeras I/DNA complex http://www.pdb.org/ pdb/explore/explore. do?structureld=1a35

http://www.pdb.org/ pdb/explore/explore. do?structureld=1 plq

http://www.pdb.org/ pdb/explore/explore. do? structureld=2vl6
Publicly available at RCSB Protein Data Bank.

Publicly available at RCSB Protein Data Bank.

Publicly available at RCSB Protein Data Bank. http://www.pdb.org/ pdb/explore/explore. do?structureld=4fdg
Publicly available at RCSB Protein Data Bank.

\section{References}

Abrahams JP, Leslie AG, Lutter R, Walker JE. 1994. Structure at 2.8 A resolution of F1-ATPase from bovine heart mitochondria. Nature 370:621-628. doi: 10.1038/370621a0.

Afonine PV, Grosse-Kunstleve RW, Echols N, Headd JJ, Moriarty NW, Mustyakimov M, Terwilliger TC, Urzhumtsev A, Zwart PH, Adams PD. 2012. Towards automated crystallographic structure refinement with phenix.refine. Acta Crystallographica Section D, Biological Crystallography 68:352-367. doi: 10.1107/S0907444912001308.

Aravind L, Koonin EV. 1999. DNA-binding proteins and evolution of transcription regulation in the archaea. Nucleic Acids Research 27:4658-4670. doi: 10.1093/nar/27.23.4658.

Atanassova N, Grainge I. 2008. Biochemical characterization of the minichromosome maintenance (MCM) protein of the crenarchaeote Aeropyrum pernix and its interactions with the origin recognition complex (ORC) proteins. Biochemistry 47:13362-13370. doi: 10.1021/bi801479s.

Bae B, Chen YH, Costa A, Onesti S, Brunzelle JS, Lin Y, Cann IK, Nair SK. 2009. Insights into the architecture of the replicative helicase from the structure of an archaeal MCM homolog. Structure 17:211-222. doi: 10.1016/j. str.2008.11.010.

Barry ER, Lovett JE, Costa A, Lea SM, Bell SD. 2009. Intersubunit allosteric communication mediated by a conserved loop in the MCM helicase. Proceedings of the National Academy of Sciences of USA 106:1051-1056. doi: 10.1073/pnas.0809192106.

Barry ER, McGeoch AT, Kelman Z, Bell SD. 2007. Archaeal MCM has separable processivity, substrate choice and helicase domains. Nucleic Acids Research 35:988-998. doi: 10.1093/nar/gkl1117.

Bochman ML, Schwacha A. 2007. Differences in the single-stranded DNA binding activities of MCM2-7 and MCM467: MCM2 and MCM5 define a slow ATP-dependent step. The Journal of Biological Chemistry 282:33795-33804. doi: 10.1074/jbc.M703824200.

Bochman ML, Schwacha A. 2008. The Mcm2-7 complex has in vitro helicase activity. Molecular Cell 31:287-293. doi: 10.1016/j.molcel.2008.05.020.

Brewster AS, Wang G, Yu X, Greenleaf WB, Carazo JM, Tjajadia M, Klein MG, Chen XS. 2008. Crystal structure of a near-full-length archaeal MCM: functional insights for an AAA+ hexameric helicase. Proceedings of the National Academy of Sciences of USA 105:20191-20196. doi: 10.1073/pnas.0808037105.

Brunger AT. 2007. Version 1.2 of the Crystallography and NMR system. Nature Protocols 2:2728-2733. doi: 10.1038/nprot.2007.406.

Brunger AT, Adams PD, Clore GM, Delano WL, Gros P, Grosse-Kunstleve RW, Jiang JS, Kuszewski J, Nilges M, Pannu NS, Read RJ, Rice LM, Simonson T, Warren GL. 1998. Crystallography \& NMR system: a new software suite for macromolecular structure determination. Acta Crystallographica Section D, Biological Crystallography 54:905-921. doi: 10.1107/S0907444998003254.

Chen YJ, Yu X, Kasiviswanathan R, Shin JH, Kelman Z, Egelman EH. 2005. Structural polymorphism of Methanothermobacter thermautotrophicus MCM. Journal of Molecular Biology 346:389-394. doi: 10.1016/j. jmb.2004.11.076.

Chong JP, Hayashi MK, Simon MN, Xu RM, Stillman B. 2000. A double-hexamer archaeal minichromosome maintenance protein is an ATP-dependent DNA helicase. Proceedings of the National Academy of Sciences of USA 97:1530-1535. doi: 10.1073/pnas.030539597.

Costa A, Ilves I, Tamberg N, Petojevic T, Nogales E, Botchan MR, Berger JM. 2011. The structural basis for MCM2-7 helicase activation by GINS and Cdc45. Nature Structural \& Molecular Biology 18:471-477. doi: 10.1038/nsmb.2004.

Costa A, Pape T, Van Heel M, Brick P, Patwardhan A, Onesti S. 2006. Structural basis of the Methanothermobacter thermautotrophicus MCM helicase activity. Nucleic Acids Research 34:5829-5838. doi: 10.1093/nar/gkl708.

Coster G, Frigola J, Beuron F, Morris EP, Diffley JF. 2014. Origin licensing requires ATP binding and hydrolysis by the mcm replicative helicase. Molecular Cell 55:666-677. doi: 10.1016/j.molcel.2014.06.034.

Cowtan K. 1994. 'dm': An automated procedure for phase improvement by density modification. Joint CCP4 and ESF-EACBM Newsletter on Protein Crystallography 31:34-38.

Duderstadt KE, Berger JM. 2013. A structural framework for replication origin opening by AAA+ initiation factors. Current Opinion in Structural Biology 23:144-153. doi: 10.1016/j.sbi.2012.11.012. 
Emsley P, Cowtan K. 2004. Coot: model-building tools for molecular graphics. Acta Crystallographica Section D, Biological Crystallography 60:2126-2132. doi: 10.1107/S0907444904019158.

Enemark EJ, Joshua-Tor L. 2006. Mechanism of DNA translocation in a replicative hexameric helicase. Nature 442:270-275. doi: 10.1038/nature04943.

Erzberger JP, Berger JM. 2006. Evolutionary relationships and structural mechanisms of AAA+ proteins. Annual Review of Biophysics and Biomolecular Structure 35:93-114. doi: 10.1146/annurev.biophys.35.040405.101933.

Evrin C, Clarke P, Zech J, Lurz R, Sun J, Uhle S, Li H, Stillman B, Speck C. 2009. A double-hexameric MCM2-7 complex is loaded onto origin DNA during licensing of eukaryotic DNA replication. Proceedings of the National Academy of Sciences of USA 106:20240-20245. doi: 10.1073/pnas.0911500106.

Fletcher RJ, Bishop BE, Leon RP, Sclafani RA, Ogata CM, Chen XS. 2003. The structure and function of MCM from archaeal M. Thermoautotrophicum. Nature Structural Biology 10:160-167. doi: 10.1038/nsb893.

Froelich CA, Kang S, Epling LB, Bell SP, Enemark EJ. 2014. A conserved MCM single-stranded DNA binding element is essential for replication initiation. eLife 3:e01993. doi: 10.7554/eLife.01993.

Fu YV, Yardimci H, Long DT, Ho TV, Guainazzi A, Bermudez VP, Hurwitz J, Van Oijen A, Scharer OD, Walter JC. 2011. Selective bypass of a lagging strand roadblock by the eukaryotic replicative DNA helicase. Cell 146: 931-941. doi: 10.1016/j.cell.2011.07.045.

Gomez-Llorente Y, Fletcher RJ, Chen XS, Carazo JM, San Martin C. 2005. Polymorphism and double hexamer structure in the archaeal minichromosome maintenance (MCM) helicase from Methanobacterium thermoautotrophicum. The Journal of Biological Chemistry 280:40909-40915. doi: 10.1074/jbc.M509760200.

Hardy CF, Dryga O, Seematter S, Pahl PM, Sclafani RA. 1997. mcm5/cdc46-bob1 bypasses the requirement for the S phase activator Cdc7p. Proceedings of the National Academy of Sciences of USA 94:3151-3155. doi: 10.1073/pnas.94.7.3151.

Hendrickson WA, Horton JR, Lemaster DM. 1990. Selenomethionyl proteins produced for analysis by multiwavelength anomalous diffraction (MAD): a vehicle for direct determination of three-dimensional structure. The EMBO Journal 9:1665-1672.

Ilves I, Petojevic T, Pesavento JJ, Botchan MR. 2010. Activation of the MCM2-7 helicase by association with Cdc45 and GINS proteins. Molecular Cell 37:247-258. doi: 10.1016/j.molcel.2009.12.030.

Jenkinson ER, Chong JP. 2006. Minichromosome maintenance helicase activity is controlled by N-and C-terminal motifs and requires the ATPase domain helix-2 insert. Proceedings of the National Academy of Sciences of USA 103:7613-7618. doi: 10.1073/pnas.0509297103.

Kainov DE, Mancini EJ, Telenius J, Lisal J, Grimes JM, Bamford DH, Stuart DI, Tuma R. 2008. Structural basis of mechanochemical coupling in a hexameric molecular motor. The Journal of Biological Chemistry 283: 3607-3617. doi: 10.1074/jbc.M706366200.

Kang S, Warner MD, Bell SP. 2014. Multiple functions for Mcm2-7 ATPase motifs during replication initiation. Molecular Cell 55:655-665. doi: 10.1016/j.molcel.2014.06.033.

Kleywegt GJ. 1996. Use of non-crystallographic symmetry in protein structure refinement. Acta Crystallographica Section D, Biological Crystallography 52:842-857. doi: 10.1107/S0907444995016477.

Kleywegt GJ. 1997. Validation of protein models from Calpha coordinates alone. Journal of Molecular Biology 273:371-376. doi: 10.1006/jmbi.1997.1309.

Kraulis PJ. 1991. Molscript - a program to produce both detailed and schematic plots of protein structures. Journal of Applied Crystallography 24:946-950. doi: 10.1107/S0021889891004399.

Krishna TS, Kong XP, Gary S, Burgers PM, Kuriyan J. 1994. Crystal structure of the eukaryotic DNA polymerase processivity factor PCNA. Cell 79:1233-1243. doi: 10.1016/0092-8674(94)90014-0.

Labib K. 2010. How do Cdc7 and cyclin-dependent kinases trigger the initiation of chromosome replication in eukaryotic cells? Genes \& Development 24:1208-1219. doi: 10.1101/gad.1933010.

Laskowski RA, Macarthur MW, Moss DS, Thornton JM. 1993. Procheck - a program to check the stereochemical quality of protein structures. Journal of Applied Crystallography 26:283-291. doi: 10.1107/S0021889892009944.

Lee SJ, Syed S, Enemark EJ, Schuck S, Stenlund A, Ha T, Joshua-Tor L. 2014. Dynamic look at DNA unwinding by a replicative helicase. Proceedings of the National Academy of Sciences of USA 111:E827-E835. doi: 10.1073/ pnas.1322254111.

Liu W, Pucci B, Rossi M, Pisani FM, Ladenstein R. 2008. Structural analysis of the Sulfolobus solfataricus MCM protein N-terminal domain. Nucleic Acids Research 36:3235-3243. doi: 10.1093/nar/gkn183.

McCoy AJ, Grosse-Kunstleve RW, Adams PD, Winn MD, Storoni LC, Read RJ. 2007. Phaser crystallographic software. Journal of Applied Crystallography 40:658-674. doi: 10.1107/S0021889807021206.

McGeoch AT, Trakselis MA, Laskey RA, Bell SD. 2005. Organization of the archaeal MCM complex on DNA and implications for the helicase mechanism. Nature Structural \& Molecular Biology 12:756-762. doi: 10.1038/ nsmb974.

Merritt EA, Bacon DJ. 1997. Raster3D: photorealistic molecular graphics. Methods in Enzymology 277:505-524. doi: 10.1016/S0076-6879(97)77028-9.

Moreau MJ, McGeoch AT, Lowe AR, Itzhaki LS, Bell SD. 2007. ATPase site architecture and helicase mechanism of an archaeal MCM. Molecular Cell 28:304-314. doi: 10.1016/j.molcel.2007.08.013.

Mossessova E, Lima CD. 2000. Ulp1-SUMO crystal structure and genetic analysis reveal conserved interactions and a regulatory element essential for cell growth in yeast. Molecular Cell 5:865-876. doi: 10.1016/ S1097-2765(00)80326-3.

Moyer SE, Lewis PW, Botchan MR. 2006. Isolation of the Cdc45/Mcm2-7/GINS (CMG) complex, a candidate for the eukaryotic DNA replication fork helicase. Proceedings of the National Academy of Sciences of USA 103:10236-10241. doi: 10.1073/pnas.0602400103. 
Neuwald AF, Aravind L, Spouge JL, Koonin EV. 1999. AAAt: a class of chaperone-like ATPases associated with the assembly, operation, and disassembly of protein complexes. Genome Research 9:27-43.

Otwinowski Z, Minor W. 1997. Processing of X-ray diffraction data collected in oscillation mode. Macromolecular Crystallography, Part A 276:307-326. doi: 10.1016/S0076-6879(97)76066-X.

Pape T, Meka H, Chen S, Vicentini G, Van Heel M, Onesti S. 2003. Hexameric ring structure of the full-length archaeal MCM protein complex. EMBO Reports 4:1079-1083. doi: 10.1038/sj.embor.embor7400010.

Pucci B, De Felice M, Rocco M, Esposito F, De Falco M, Esposito L, Rossi M, Pisani FM. 2007. Modular organization of the Sulfolobus solfataricus mini-chromosome maintenance protein. The Journal of Biological Chemistry 282:12574-12582. doi: 10.1074/jbc.M610953200.

Pucci B, De Felice M, Rossi M, Onesti S, Pisani FM. 2004. Amino acids of the Sulfolobus solfataricus minichromosome maintenance-like DNA helicase involved in DNA binding/remodeling. The Journal of Biological Chemistry 279:49222-49228. doi: 10.1074/jbc.M408967200.

Redinbo MR, Stewart L, Kuhn P, Champoux JJ, Hol WG. 1998. Crystal structures of human topoisomerase I in covalent and noncovalent complexes with DNA. Science 279:1504-1513. doi: 10.1126/science.279.5356.1504.

Remus D, Beuron F, Tolun G, Griffith JD, Morris EP, Diffley JF. 2009. Concerted loading of Mcm2-7 double hexamers around DNA during DNA replication origin licensing. Cell 139:719-730. doi: 10.1016/j.cell.2009.10.015.

Remus D, Diffley JF. 2009. Eukaryotic DNA replication control: lock and load, then fire. Current Opinion in Cell Biology 21:771-777. doi: 10.1016/j.ceb.2009.08.002.

Sakakibara N, Kasiviswanathan R, Melamud E, Han M, Schwarz FP, Kelman Z. 2008. Coupling of DNA binding and helicase activity is mediated by a conserved loop in the MCM protein. Nucleic Acids Research 36: 1309-1320. doi: 10.1093/nar/gkm1160.

Schrodinger LLC. 2010. The PyMOL molecular graphics system, Version 1.3r1.

Sheu YJ, Stillman B. 2006. Cdc7-Dbf4 phosphorylates MCM proteins via a docking site-mediated mechanism to promote S phase progression. Molecular Cell 24:101-113. doi: 10.1016/j.molcel.2006.07.033.

Sheu YJ, Stillman B. 2010. The Dbf4-Cdc7 kinase promotes S phase by alleviating an inhibitory activity in Mcm4. Nature 463:113-117. doi: 10.1038/nature08647.

Singleton MR, Dillingham MS, Wigley DB. 2007. Structure and mechanism of helicases and nucleic acid translocases. Annual Review of Biochemistry 76:23-50. doi: 10.1146/annurev.biochem.76.052305.115300.

Slaymaker IM, Fu Y, Toso DB, Ranatunga N, Brewster A, Forsburg SL, Zhou ZH, Chen XS. 2013. Minichromosome maintenance complexes form a filament to remodel DNA structure and topology. Nucleic Acids Research 41:3446-3456. doi: 10.1093/nar/gkt022.

Terwilliger TC. 2000. Maximum-likelihood density modification. Acta Crystallographica Section D, Biological Crystallography 56:965-972. doi: 10.1107/S0907444900005072.

Terwilliger TC. 2004. Using prime-and-switch phasing to reduce model bias in molecular replacement. Acta Crystallographica Section D, Biological Crystallography 60:2144-2149. doi: 10.1107/S0907444904019535.

Thomsen ND, Berger JM. 2009. Running in reverse: the structural basis for translocation polarity in hexameric helicases. Cell 139:523-534. doi: 10.1016/j.cell.2009.08.043.

Vagin AA, Steiner RA, Lebedev AA, Potterton L, Mcnicholas S, Long F, Murshudov GN. 2004. REFMAC5 dictionary: organization of prior chemical knowledge and guidelines for its use. Acta Crystallographica Section D, Biological Crystallography 60:2184-2195. doi: 10.1107/S0907444904023510.

Yardimci H, Loveland AB, Habuchi S, Van Oijen AM, Walter JC. 2010. Uncoupling of sister replisomes during eukaryotic DNA replication. Molecular Cell 40:834-840. doi: 10.1016/j.molcel.2010.11.027.

Yoshimochi T, Fujikane R, Kawanami M, Matsunaga F, Ishino Y. 2008. The GINS complex from Pyrococcus furiosus stimulates the MCM helicase activity. The Journal of Biological Chemistry 283:1601-1609. doi: 10.1074/jbc. M707654200. 